\title{
Dietary Antioxidant An Indispensible Nutrient
}

\author{
Shizma Junejo, Zara Sami, Faiza Rafique Shaikh
}

Free radicals prevail as atoms, molecules or unpaired electrons being extremely reactive as well as unstable ${ }^{1}$. They can lead to chemical reactions alongside different molecules. Having been originated from constituents like oxygen, sulphur as well as nitrogen which contribute towards production of reactive oxygen species (ROS), reactive nitrogen species (RNS), reactive sulphur species $(\mathrm{RSS})^{1}$. They include superoxide anion $\left(\mathrm{O}_{2}-\bullet\right)$, hydroperoxyl radical $\left(\mathrm{HO}_{2} \bullet\right)$, hydroxyl radical $(\bullet \mathrm{OH})$, hydrogen peroxide $\left(\mathrm{H}_{2} \mathrm{O}_{2}\right)$, singlet oxygen $(1$ $\mathrm{O}_{2}$ ), hypochlorous acid $(\mathrm{HOCl})$ and peroxynitrite (ONOO). Free radical species are formed in the course of plentiful oxidative metabolic processes in mitochondria ${ }^{1}$.

There is extremely mild equilibrium between the making together with the counteraction of ROS through antioxidants. As a consequence whenever the equilibrium gravitates towards over production of ROS then as a sequel to oxidative stress cells begin deteriorating.

Being usual component of metabolism inside mitochondria, by way of xanthine oxidase, peroxisomes, inflammation processes, phagocytosis, arachidonate pathways, ischemia and physical exercise, free radicals production occurs inwardly ${ }^{3}$. Assisting with advanced making of free radicals are extraneous causes including smoking, environmental pollutants, drugs, pesticides, industrial solvents as well as ozone $^{2}$.

Free radicals exist as either atoms, molecules, ions inclusive of unpaired electrons which are extremely unstable plus in addition efficacious in relation to chemical reactions alongside other molecules ${ }^{3}$. Emanation of free radicals is from three elements including oxygen, nitrogen as well as sulfur, hence forming reactive oxygen species (ROS), reactive nitrogen species (RNS) as well as reactive sulfur species (RSS). ROS consist of free radicals such as superoxide anion $\left(\mathrm{O}_{2}-\bullet\right)$, hydroperoxyl radical $\left(\mathrm{HO}_{2} \bullet\right)$, hydroxyl radical $(\bullet \mathrm{OH})$, nitric oxide (NO) plus other species such as hydrogen peroxide $\left(\mathrm{H}_{2} \mathrm{O}_{2}\right)$, singlet oxygen $\left(1 \mathrm{O}_{2}\right)$, hypochlorous acid $(\mathrm{HOCl})$ and peroxynitrite (ONOO-) ${ }^{3}$. Free radicals, antioxidants as well

Shizma Junejo
Senior Lecturer, Department of Biochemistry
Bahria University Medical and Dental College Karachi
Email: shizjunejo@hotmail.com
Zara Sami
Senior Lecturer, Department of Biochemistry
Bahria University Medical and Dental College Karachi
Faiza Rafique Shaikh
Senior Lecturer, Department of Biochemistry
Bahria University Medical and Dental College Karachi
Received: 20-11-18
Accepted: 12-12-18

as co-factors prevail as three predominant spheres which presumably may be partly responsible for aging process retardment ${ }^{1}$.

DNA is also affected with free radicals attack. Changes are both chemical and structural. They are deletions, frame shifts, strand breaks, chromosomal arrangements, amendments of all bases, formation of base-free sites ${ }^{4}$.

Peroxidation of lipid is begun through advancement toward side chain of fatty acid via a free radical directed towards separating a hydrogen atom from a methylene carbon ${ }^{5}$. Higher number of double bonds, makes it additionally simple to remove hydrogen atoms to form a radical and produce monounsaturated fatty acids and saturated fatty acids. The reaction continues with the formation of peroxyl radical that dislodges further hydrogen atoms and completes the process of lipid peroxidation ${ }^{5}$

There are two chief classes of antioxidants. These include enzymatic antioxidants as well as non-enzymatic antioxidants. In addition the enzymatic antioxidants exist as grouped into primary as well as secondary enzymatic defenses ${ }^{5}$.Three important enzymes constitute primary defence and their function is controlling formation of free radicals. These include glutathione peroxidase, which provides two electrons reducing peroxides through formation of selenols, catalase which turns hydrogen peroxide to water plus molecular oxygen. Also superoxide dismutase, which changes superoxide anions towards hydrogen peroxide ${ }^{1}$. As part of secondary enzymatic defense, the enzymes are glutathione reductase as well as glucose-6-phosphate dehydrogenase ${ }^{6}$. Glutathione reductase reduces glutathione an antioxidant against oxidized state of it, towards reduced form of it $^{6}$. Glucose-6-phosphate reconstructs NADPH, leading to reducing environment ${ }^{6}$. The group of non-enzymatic antioxidants includes vitamins such as A, E, C, enzyme cofactors (Q10), minerals like zinc and selenium, peptides like glutathione, phenolic acids as well as nitrogen compounds like uric acid ${ }^{5}$.

The antioxidants are playing major role in human health and immune system, as they are maintaining function and structure of cell by means of destruction of free radicals ${ }^{7}$.Carotenoids and tocopherol in combination giving more potent antioxidant effect on biological membranes the lipid bilayers ${ }^{7}$.Carotenoids detoxify nitrous oxide, sulfonyl, thiyl, peroxyl and single oxygen and save the lipid peroxidation from hydroxyl and superoxide free radicals ${ }^{7}$.Optimum nutrient content allows efficacious absorption as well as metabolism of antioxidants in food ${ }^{7}$. Reactive oxygen species are formed by partial reduction of molecular oxygen as a result of aerobic metabolism through 
the reaction with drugs and environmental toxins ${ }^{8}$. They produce dangerous chemical outcomes effecting DNA, lipids, proteins and lead to death of cell. Oxidative stress is a condition which is produced due to excess of free radicals and conversely as a result of diminished antioxidant ${ }^{8}$. Membrane lipid peroxidation causes membrane fragility leading to anemia which is hemolytic ${ }^{9}$. Effect of reactive oxygen species on lipids, proteins, carbohydrates plus DNA culminates in numerous diseases, rheumatoid arthritis, reperfusion injury, atherosclerosis and lung diseases ${ }^{10}$.

Tissue defense mechanism against free-radical damage generally includes vitamin $\mathrm{C}$, vitamin $\mathrm{E}$ and â-carotene and other carotenoids as the major vitamin antioxidant sources ${ }^{5}$. In addition, several metalloenzymes which include glutathione peroxidase (Se), catalase (Fe) and superoxide dismutase $(\mathrm{Cu}, \mathrm{Zn}$, and $\mathrm{Mn})$ are also critical in protecting the internal cellular constituents from oxidative damage ${ }^{5}$.

It is extensively established that a plant-based diet incorporating increased indulgence in consuming fruits, vegetables as well as several loaded with nutrient plantbased foods can lower the risk of diseases related to oxidative stress. ${ }^{11}$ Natural antioxidant molecules have been suggested as a flipside mode of assistance for the prevention of agerelated neurological diseases ${ }^{12}$. Various types of antioxidant molecules such as polyphenols and carotenoids as well as long-established antioxidant vitamins, vitamin $\mathrm{C}$ and $\mathrm{E}$ can enrich with this prevention ${ }^{12}$. According to epidemiological studies significant differences exist in the prevalence of various diseases among communal groups which have distinctive food practices. As an instance, epidemiological evidence has revealed that the Mediterranean diet, that has ample antioxidants, has an efficacious role in the prevention of age-related diseases like Alzheimer's. Green tea polyphenols are considered as strong antioxidants countering hydroxyl radicals, nitric oxide and lipid oxidation ${ }^{12}$.

The dietary antioxidants like ascorbates, tocopherols as well as carotenoids are acknowledged. Furthermore, superfluous reporting exists concerning role of these in health. Vitamin $\mathrm{C}$, vitamin $\mathrm{E}$, along with betacarotene plus additional carotenoids as well as oxycarotenoids, including lycopene, lutein are amongst maximally extensively investigated antioxidants from diet. ${ }^{13}$ Vitamin $\mathrm{C}$ has been studied as an exceptionally significant water-soluble antioxidant within extracellular fluids ${ }^{13}$. It has a role in counteracting reactive oxygen species within aqueous state prior to peroxidation of lipid. An essential soluble in lipid antioxidant is vitamin E. It is extremely potent chain-breaking antioxidant being associated with cell membrane, shielding fatty acids in membrane against lipid peroxidation ${ }^{13}$. Tissues upscale in lipid derive antioxidant protection through beta carotene a well as additional carotenoids ${ }^{13}$.

Consumption of $250 \mathrm{~g}$ of strawberries by healthy participants was correlated to reasonable yet important antioxidant activity elevation within serum as revealed by an evaluation, exhibiting possibility of boosting defense of body as counter to long-standing disease ${ }^{14}$. Antioxidants can prevail as synthetic or natural. Various sources of natural antioxidants include legumes, nuts, oilseeds, cereals, fruits, vegetables, animal products ${ }^{15}$. Adverse free radicals, having involvement in highly frequent cancers as well as distinct degenerative diseases inclusive of inadequate brain activity are scavenged by antioxidants ${ }^{15}$.

Chief sources of naturally occurring antioxidants include fruits, vegetables, whole grains, green and black tea, coffee ${ }^{15}$. Antioxidants from oilseeds are represented by flaxseed, sunflowers, soybean, cottonseed and canola antioxidants. Fruits include various vitamins and mineral salts as well as dietary fiber ${ }^{15}$. Nearly all fruits constitute a sufficient way of providing vitamin $\mathrm{C}$, carotenoids as well as polyphenolic compounds ${ }^{15}$. As proclaimed, extremely powerful antioxidant activity is linked to apples, restricting cancer cell propagation, lowering lipid oxidation, in addition decreasing cholesterol. As researched potato provides an adequate supply of antioxidants such as ascorbic acid, á-tocopherol and polyphenolic compounds ${ }^{15}$.

Vegetables as well as fruits constitute the predominant source of flavonoids ${ }^{15}$. It has been revealed that pumpkin seed extract has anticancer, antimutagenic as well as antioxidant activities $^{15}$. According to sufficient research black pepper as in piper nigrum seeds exhibit antioxidant plus radical scavenging activities. The finest sources of antioxidants from amongst vegetables are tomatoes, red pepper, brassica vegetables, onion, garlic and red beet ${ }^{15}$.

Antioxidants can be treated as healthy compounds as advocated. It has been revealed by surveys that natural antioxidants exhibit exceptional role in humans, in addition they are free from harm ${ }^{15}$. Hence, we should use them in our diet. Accordingly use of these compounds as well as products assuredly influences our own health, as a consequence they must be utilized by us in our food.

\section{REFERENCES:}

1. Rahman, K. Studies on free radicals, antioxidants and cofactors. Clin.Inverv. Aging.2007; 2:219-236.

2. Wiernsperger N.F. Oxidative stress as a therapeutic target in diabetes: Revisiting the controversy. Diabetes Metab.2003; 29:579-585.

3. Lü J, Lin P.H, Yao Q, Chen C. Chemical and molecular mechanisms of antioxidants: Experimental approaches and model systems. J. Cell Mod. Med. 2010;14:840-60.

4. Lobo V, Phatak A, Chandra N. Free radicals and functional foods: Impact on human health. Pharmacogn. Rev. 2010;4:11826.

5. Carocho $\mathrm{M}$ and Ferreira ICFR."A Review on Antioxidants, Prooxidants and Related Controversy: Natural and synthetic compounds. Screening and Analysis Methodologies and Future Perspectives," Food and Chemical Toxicology.2013;51:1525. 
6. Ratnam DV, Ankola DD, Bhardwaj V, Sahana DK and Kumar MNVR. "Role of Antioxidants in Prophylaxis and Therapy: A Pharmaceutical Perspective". Journal of Controlled Release.2006;113(3):189- 207.

7. McDowell LR, Wilkinson N, Madison R and Felix T. Vitamins and minerals functioning as antioxidants with supplementation considerations. Florida Ruminant Nutrition Symposium. 2007.

8. Ferrier DR. Lippincott's Illustrated Reviews: Biochemistry. 6th ed. Lipincott Williams and Wilkins. P.no 143).

9. Murray RK,Bender DA, Botham KM, Kennelly PJ, Rodwell VW and Weil PA. Harper's Illustrated Biochemistry. 29th Edition. McGrawHill.Pg no 532.

10. Zadak Z, Hyspler R, Ticha A, Hronek M, Fikrova P, Rathosuka $\mathrm{J}$, et al. Antioxidants and vitamins in Clinical Conditions. Physiol Res. 2009;58(1):13-17.

11. Carlsen MH, Halvorsen BL, Holte K, Bohn SK, Dragland $\mathrm{S}$, Sampson L, et al. The total antioxidant content of more than 3100 foods, beverages, spices, herbs and supplements used worldwide. Nutrition Journal. 2010;9(3):1-11.

12. Albarracin SL, Stab B, Casas Z, Sutachan JJ, Samudio I, Gonzalez J. Effects of natural antioxidants in neurodegenerative disease. Nutritional Neuroscience. 2012:15(1);1-9.

13. Yadav A, Kumari R, Yadav A, Mishra J.P, Srivatva S and Prabha S. Antioxidants and its functions in human body - A Review. Research in Environment and Life Sciences. 2016;9(11) :1328-1331.

14. Henning SM, NP, Zhang Y, Li L, Gao K, Lee RP. Et al. Strawberry Consumption Is Associated with Increased Antioxidant Capacity in Serum. J Med Food. 2010;13(1): 116-122.

15. Akbarirad H, Ardabili GA, Kazemeini, S M and Khaneghah MA. An overview on some of important sources of natural antioxidants.International Food Research Journal. 2016;23(3): 928-933. 